\title{
Molecular Switches at Surfaces
}

\author{
Martin Weinelt and Felix von Oppen \\ Freie Universität Berlin, Fachbereich Physik, Arnimallee 14, 14195 Berlin, Germany \\ E-mail: weinelt@physik.fu-berlin.de
}

In nature, molecules exploit the interaction with their environment to realize complex functionalities on the nanometer length scale. Physical, chemical and/or biological specificity is frequently achieved by switching of molecules between microscopically different states. Paradigmatic examples are the energy production in proton pumps of bacteria or the signal conversion in human vision which rely on switching molecules between different configurations or conformations by external stimuli.

The remarkable reproducibility and unparalleled fatigue resistance of these natural processes make it highly desirable to emulate nature and develop artificial systems with molecular functionalities. A promising avenue towards this goal is to anchor the molecular switches at surfaces, offering new pathways to control their functional properties, to apply electrical contacts, or to integrate switches into larger systems. Anchoring at surfaces allows one to access the full range from individual molecular switches to self-assembled monolayers of well-defined geometry and to customize the coupling between molecules and substrate or between adsorbed molecules. Progress in this field requires both synthesis and preparation of appropriate molecular systems and control over suitable external stimuli, including light, heat, or electrical currents. To optimize switching and to generate function, it is essential to unravel the geometric structure, the electronic properties, and the dynamic interactions of the molecular switches on surfaces.

The present special issue entitled Molecular Switches at Surfaces collects 18 contributions describing different aspects of this research field. They analyze elementary processes, both in single molecules and in ensembles of molecules, which involve molecular switching and concomitant changes of optical, electronic, or magnetic properties. Two topical reviews summarize the current status, including both challenges and achievements, in the field of molecular switches on metal surfaces, focusing on electronic and vibrational spectroscopy in one case and scanning tunneling microscopy studies in the other. Original research articles describe results on many aspects of the field, including:

- Self-assembly, self-organization, and controlled growth of molecular layers 
on various substrates.- Highly-ordered arrays provide model systems with extraordinary structural properties, allow one to adjust interactions between molecules and between molecule and substrate, and can be robustly prepared from solution, an essential prerequisite for applications.

- Conformational or electronic switching of molecules adsorbed at metal and semiconductor surfaces.- These studies highlight the elementary processes governing molecular switching at surfaces as well as the wide range of possible stimuli.

- Carbon-based substrates such as graphene or carbon nanotubes.- These substrates are attractive by their effective two-dimensionality which implies that switching of adsorbed molecules can effect a significant backaction on the substrate.

- Mechanisms of conformational switching.-- Several contributions study the role of electron-vibron coupling and heating in current-induced conformational switching.

We hope that the presented collection of articles will stimulate and encourage researchers in surface physics and interfacial chemistry to contribute to the still emerging field of molecular switches at surfaces. We wish to acknowledge the support of and the input from many colleagues in preparing this special issue. A significant fraction of this work has been conducted in the framework of the Sonderforschungsbereich 658 Elementary Processes in Molecular Switches at Surfaces of the Deutsche Forschungsgemeinschaft to which we are grateful for financial support. 